\title{
Pentraxin 3 as a new indicator of cardiovascular-related death in patients with advanced chronic kidney disease
}

\author{
Marcin Krzanowski ${ }^{1}$, Katarzyna Krzanowska ${ }^{1}$, Mariusz Gajda ${ }^{2}$, Paulina Dumnicka ${ }^{3}$, \\ Artur Dziewierz ${ }^{4}$, Karolina Woziwodzka', Jan A. Litwin², Władysław Sułowicz ${ }^{1}$ \\ 1 Department of Nephrology, Jagiellonian University Medical College, Kraków, Poland \\ 2 Department of Histology, Jagiellonian University Medical College, Kraków, Poland \\ 3 Department of Medical Diagnostics, Jagiellonian University Medical College, Kraków, Poland \\ 4 2nd Department of Cardiology, Jagiellonian University Medical College, Kraków, Poland
}

\section{KEY WORDS}

cardiovascular mortality, chronic kidney disease, common carotid artery intima-media thickness, pentraxin 3 , radial artery calcification
Correspondence to:

Marcin Krzanowski, MD, PhD, Katedra

i Klinika Nefrologii, Uniwersytet Jagielloński, Collegium Medicum, ul. Kopernika 15c, 31-501 Kraków Poland, phone: +48124247800 e-mail:mkrzanowski@op.pl Received: November 2, 2016. Revision accepted: February 27, 2017 Published online: February 27, 2017 Conflict of interests: none declared. Pol Arch Intern Med. 2017:

127 (3): 170-177 doi:10.20452/pamw.3944 Copyright by Medycyna Praktyczna Kraków 2017

\section{ABSTRACT}

INTRODUCTION Pentraxin 3 (PTX3) is involved in inflammatory response by recognizing pathogens and damaged tissues.

OBJECTIVES The aim of this study was to assess the relationship between PTX3 levels and all-cause and cardiovascular (CV) mortality in patients with chronic kidney disease (CKD) during 5-year follow-up. PATIENTS AND METHODS The study included 78 patients (51 on hemodialysis and 27 on predialysis). We measured the levels of PTX3, calcium, phosphate, intact parathyroid hormone, high-sensitivity Creactive protein (hs-CRP), interleukin 6 (IL-6), fibroblast growth factor 23 (FGF-23), osteopontin (OPN), osteocalcin (OC), osteoprotegerin (OPG), fetuin A, tumor necrosis factor receptor 2 (TNFR2), transforming growth factor $\beta_{1}$ (TGF- $\left.\beta_{1}\right)$, hepatocyte growth factor (HGF), stromal cell-derived factor $1 \alpha$ (SDF-1 $\alpha$ ), and thrombomodulin (TM). In a subgroup of 45 patients, fragments of the radial artery obtained during creation of hemodialysis access were stained for calcifications. In 51 patients, ultrasonography was performed to assess common carotid artery intima-media thickness (CCA-IMT).

RESULTS The median serum concentrations of PTX3 were $1.43 \mathrm{ng} / \mathrm{ml}$ (interquartile range, 0.74-2.50). Higher concentrations of fibrinogen, hs-CRP, IL-6, TNFR2, TGF- $\beta_{1}$, HGF, OPN, OPG, FGF-23, TM, and SDF-1 $\alpha$ and lower albumin and uric acid levels were observed in patients with PTX3 above the median. During follow-up, 27 patients (35\%) died, including 25 due to CV causes. In contrast to hs-CRP levels, baseline PTX3 levels predicted CV mortality independently of classic CV risk factors. PTX3 levels also significantly predicted mortality after adjustment for age, baseline dialysis status, serum OPG and CRP levels, radial artery calcifications, and CCA-IMT.

CONCLUSIONS We postulate that PTX3 might be an early marker of CV mortality in patients with advanced CKD, yet before the increase in the levels of a specific marker for systemic inflammation such as hs-CRP.

INTRODUCTION Vascular calcifications are the most important risk factor in the pathogenesis of cardiovascular diseases (CVDs) in patients with chronic kidney disease (CKD). In addition, the presence of endothelial dysfunction is associated with inflammation, which has repeatedly been shown to enhance the risk for cardiovascular (CV) mortality both in renal and nonrenal populations.
Pentraxin 3 (PTX3) is a novel acute-phase reactant produced by various cells including monocytes/macrophages, neutrophils, vascular smooth muscle cells, fibroblasts, and endothelial cells. Importantly, PTX3 levels correlate with the risk of vascular events. ${ }^{1-3}$ PTX3 is synthesized locally at the inflammatory sites and may represent a marker of local inflammation at sites of vessel injury. PTX3 has additional regulatory functions, including effects on angiogenesis, 
atherosclerotic lesion development, apoptotic cell clearance, tissue repair, and regulation of renal immunopathology. ${ }^{4}$

PTX3 is a secreted glycoprotein belonging to the pentraxin family. The molecule is so called long pentraxin, structurally related to, but distinct from, classic short pentraxins, such as C-reactive protein (CRP) and serum amyloid P. However, unlike CRP, which is synthesized mainly in the liver, PTX3 is synthesized at the site of inflammation.

PTX3-positive neutrophils infiltrate atherosclerotic plaques, suggesting that PTX3 derived from neutrophils (and also from macrophages) plays an important role in atherogenesis. ${ }^{5}$

Elevated systemic PTX3 levels appear to be a powerful marker of inflammatory status, better than CRP, and a superior outcome predictor in patients with $\mathrm{CKD} .{ }^{6-8}$ Sjoberg et $\mathrm{al}^{9}$ reported that PTX3 is a rapid and sensitive marker of inflammation in patients with CKD, and higher PTX3 levels are associated with lower glomerular filtration rate (GFR) and independently predict incident CKD in elderly patients. Zhou et $\mathrm{a}^{10}$ postulated that this marker could be a better predictor of peripheral artery disease than CRP in patients on hemodialysis (HD). Blood vessels produce large amounts of PTX3 during inflammation, and the level of circulating PTX3 increases in several pathological conditions affecting the CV system. ${ }^{5}$ The presence of aortic valve calcifications was independently associated with PTX3 levels, in addition to age, CKD, and coronary artery calcifications. Interestingly, PTX3 levels and age were independent predictors of the presence of aortic valve calcifications in patients without aortic valve stenosis. ${ }^{11}$

The aim of the present study was to assess the relationship between serum PTX3 levels and all-cause and CV mortality in patients with CKD during a 5-year follow-up. Additionally, we studied the associations between serum concentration of PTX3 and selected markers of inflammation, oxidative stress, endothelial dysfunction, and bone turnover, as well as radial artery calcification assessed histologically and common carotid artery intima-media thickness (CCA-IMT) measured by ultrasound.

PATIENTS AND METHODS Study design The study included patients with stage 5 CKD in whom arteriovenous fistula (AVF) for HD access was created for the first time, allowing collection of radial artery samples for a histological examination. Cross-sectional data were obtained immediately before the procedure and included clinical assessment of patients, CCA-IMT measurements, and assessment of laboratory parameters (markers of inflammation, oxidative stress, endothelial dysfunction, and bone turnover). Then the patients were prospectively followed for 5 years, and the data on both all-cause and CV mortality were collected.

Patients The study population consisted of 78 consecutive patients (stage $5 \mathrm{CKD}$ ) treated at the
Department of Nephrology, University Hospital, Kraków, Poland, including 51 patients on maintenance HD and 27 patients on predialysis.

At baseline, 10-year risk of death from CVD was calculated using the Polish version of the Systematic Coronary Risk Evaluation (SCORE) ${ }^{12}$ in accordance with the published guidelines. ${ }^{13}$ The CCA-IMT was assessed by ultrasound (B presentation, Acuson Siemens 128 XP/10 apparatus equipped with linear head at 5/7 MHz, Mountain View, California, United States). The measurements were performed bilaterally at $0.5 \mathrm{~cm}$ and $2 \mathrm{~cm}$ below the division of the common $\mathrm{ca}$ rotid artery during diastolic phase of the heart cycle. The results were expressed as the arithmetic means of the values obtained for the left and right arteries.

Data on mortality were collected over a 5-year period. CV mortality was defined as death due to myocardial infarction (MI), stroke, or heart failure (HF). The date and cause of death was determined on the basis of the patients' hospital records. The person who collected the data on mortality was blinded to the results of laboratory tests, histological examination, and CCA-IMT assessment.

The study was approved by the Bioethics Committee of Jagiellonian University, and all patients signed an informed consent to participate in the study.

Laboratory tests In all patients, selected biochemical parameters were measured, including serum concentrations of creatinine, glucose, intact parathyroid hormone, total calcium and phosphate, high-sensitivity CRP (hs-CRP), interleukin 6 (IL-6), PTX3, tumor necrosis factor receptor 2 (TNFR2), transforming growth factor $\beta_{1}$ (TGF- $\beta_{1}$ ), hepatocyte growth factor (HGF), thrombomodulin (TM), stromal cell-derived factor $1 \alpha$ $(\mathrm{SDF}-1 \alpha)$, osteoprotegerin (OPG), osteopontin $(\mathrm{OPN})$, osteocalcin $(\mathrm{OC})$, fibroblast growth factor 23 (FGF-23), and fetuin A. The estimated GFR (eGFR) was calculated by the Modification of Diet in Renal Disease formula: eGFR $=186 \times$ serum creatinine $(\mu \mathrm{mol} / \mathrm{l}) \times 0.0113-1.154 \times \operatorname{age}^{-0.203} \times 11$ $4 \times(0.742$ for women $) .{ }^{14}$

Blood samples were obtained at baseline, on the morning before creation of AVF for HD access. Serum samples for enzyme-linked immunosorbent assays (ELISAs) were aliquoted and stored at $-70^{\circ} \mathrm{C}$ until assayed (no longer than 3 months). Plasma samples used to assess oxidative stress parameters were protected from light, placed on ice, and centrifuged within 2 hours after collection, then aliquoted and stored at $-30^{\circ} \mathrm{C}$ until analysis (no longer than 1 month).

Routine biochemical tests were carried out using automatic biochemical analyzers: Hitachi 917 (Hitachi, Japan) and Modular P (Roche Diagnostics, Mannheim, Germany). The concentration of hs-CRP was measured using the immunonephelometric method (Nephelometer BN II, Siemens Healthcare Diagnostics, Germany). 
Inflammatory, calcification, and endothelial dysfunction markers were assessed using ELISA microplate immunoassays and ELX808 automatic reader (BIO-TEK ${ }^{\circledR}$ Instruments Inc., Vermont, Vermont, United States). The following kits were applied: IL-6, PTX3, TNFR2, TGF- $\beta_{1}$, HGF, TM, SDF- $1 \alpha$ (R\&D Systems, Minneapolis, Minnesota, United States); OPG, fetuin A (BioVendor, Brno, Czech Republic); OPN (R\&D Systems); OC (Metra/Quidel, San Diego California, United States); and FGF-23 (Immunotopics Int., San Clemente, California, United States).

Histology In 45 patients ( 27 men and 18 women; mean [SD] age, 62 [16] years; 25 patients on HD at baseline), samples of peripheral arteries were collected for histological assessment. Small fragments of the radial artery wall (approx. $5 \times 2 \mathrm{~mm}$ in size) were excised during the first creation of AVF for HD access and fixed overnight in $10 \%$ phosphate-buffered formalin. Serial $10 \mu \mathrm{m}$-thick cryosections were cut and thaw-mounted on poly-L-lysine coated slides. Sections were stained with alizarin red and were examined under an Olympus BX-50 microscope (Olympus, Tokyo, Japan). Digital images were acquired using Olympus DP-71 digital CCD camera controlled by the Olympus AnalySIS FIVE software. The extent of calcification was semiquantitatively evaluated by 2 independent observers. The degree of mineralization was classified according to the following scale: 0 , no mineral content; 1 , a few small dispersed concretions; 2, numerous small dispersed concretions; 3, larger granular concretions; and 4, large areas occupied by fused mineral deposits.

The reproducibility of the morphological analysis was confirmed by the Bland-Altman method and by calculating intraclass correlation coefficient (which was 0.88).

Statistical analysis The number of patients (percentage of the group) were reported for categories. Data were expressed as mean (SD) or median (Q1; Q3) for continuous variables, depending on the distribution (assessed by the Shapiro-Wilk test). The $\chi^{2}$ test was used for contingency tables. The $t$ test or Mann-Whitney test was employed for simple comparisons between the groups. Simple correlations were assessed using the Pearson correlation coefficient, after log-transformation of right-skewed variables. A multiple linear regression analysis was used to find independent predictors of $\log$ (PTX3). The analysis included the main confounders (age and dialysis status at baseline) and the most representative predictors chosen for each group, that is, the markers of inflammation, bone and mineral metabolism, and endothelial dysfunction. Survival time was calculated between the start date at the inclusion of a patient into the study and the date at death, renal transplantation, or the end of the study. It was estimated using the Kaplan-Meier method and compared with the log-rank test. Simple and multiple Cox proportional hazard models included PTX3 (as continuous variable) and a priori selected variables that have been associated with survival in CKD, as well as the main confounders (age and dialysis status at baseline). All tests were 2-tailed and the results were considered significant at a $P$ value of 0.05 or less. The analysis was performed with the use of Statistica 10 software (StatSoft, Tulsa, Oklahoma, United States).

RESULTS Associations between PTX3 concentrations and the markers of inflammation, endothelial dysfunction, and oxidative stress Median serum PTX3 concentrations in the studied group of 78 patients with stage $5 \mathrm{CKD}$ were $1.43 \mathrm{ng} / \mathrm{ml}(0.74$; $2.50 \mathrm{ng} / \mathrm{ml}$ ). Patients with PTX3 levels above the median had lower body mass index (BMI). They were more often on dialysis at baseline, and among those dialyzed, the treatment duration was longer (TABLE 1). As expected, patients with higher PTX3 were characterized by higher concentrations of other inflammatory markers: fibrinogen, hs-CRP, IL- 6 , TNFR2, TGF- $\beta_{1}$, and HGF, and lower albumin concentrations. Moreover, they had higher OPN, OPG, FGF-23, TM, and SDF- $1 \alpha$ levels as well as lower uric acid levels (TABLE 1).

PTX3 concentrations significantly correlated with age, BMI, dialysis treatment duration at baseline, serum creatinine and uric acid levels, other studied inflammatory markers, markers of mineral and bone metabolism, and markers of endothelial cell damage (TABLE 2). In multiple regression, $\log (\mathrm{OPG})$ significantly predicted $\log$ (PTX3) (beta [standard error]: 0.39 [0.14]; $P=0.039)$ independently of age, dialysis status at baseline, $\log (\mathrm{IL}-6)$, and $\log (\mathrm{TM})$.

Histological examination of the radial artery Mineralization of the arterial wall was identified in histological sections stained with alizarin red. Calcifications of various grades (FIGURE 1) were detected in radial artery samples of 30 patients (56\%). The mineral deposits were found almost exclusively in the vascular media.

Median serum PTX3 concentrations did not significantly differ between patients with and without radial artery calcifications: $1.39 \mathrm{ng} / \mathrm{ml}(0.74$; $2.93 \mathrm{ng} / \mathrm{ml})$ and $1.02 \mathrm{ng} / \mathrm{ml}(0.56 ; 2.27 \mathrm{ng} / \mathrm{ml})$, respectively, $P=0.2$. There was no significant association between PTX3 levels and progression of calcifications.

Common carotid artery ultrasound Data from common carotid artery ultrasound were available in 51 patients (65\%), including 33 men and 21 women (mean [SD] age, 62 [17] years), of whom 31 (57\%) were on HD at baseline. CCA-IMT values were not associated with PTX3 concentrations ( $r=0.22$; $P$ $=0.1$ ). Also, median PTX3 levels did not differ between patients with and without atherosclerotic plaques in the common carotid artery: $1.85 \mathrm{ng} / \mathrm{ml}$ $(0.78 ; 0.79)$ and $1.04 \mathrm{ng} / \mathrm{ml}(0.59 ; 1.99)$, respectively, $P=0.1$. 
TABLE 1 Clinical characteristics and laboratory data of patients with pentraxin 3 concentrations below and above the median value of $1.43 \mathrm{ng} / \mathrm{ml}$

\begin{tabular}{|c|c|c|c|}
\hline Parameter & $\begin{array}{l}\text { PTX3 } \leq 1.43 \mathrm{ng} / \mathrm{ml} \\
(\mathrm{n}=39)\end{array}$ & $\begin{array}{l}\text { PTX3 >1.43 ng/ml } \\
\quad(\mathrm{n}=39)\end{array}$ & $P$ value \\
\hline Age, y, mean (SD) & $59(16)$ & $64(17)$ & NS \\
\hline Male sex, n (\%) & $23(60)$ & $23(59)$ & NS \\
\hline Hemodialyzed, n (\%) & $20(51)$ & $31(79)$ & 0.009 \\
\hline Dialysis therapy duration ${ }^{\mathrm{a}}$, mo, median $(01 ; 03)$ & $10(3 ; 30)$ & $28(6 ; 60)$ & 0.048 \\
\hline $\mathrm{BMI}, \mathrm{kg} / \mathrm{m}^{2}$, mean $(\mathrm{SD})$ & $27.2(5.9)$ & $24.6(4.4)$ & 0.024 \\
\hline Diabetes, $\mathrm{n}(\%)$ & $11(28)$ & $15(38)$ & NS \\
\hline Hypertension, n (\%) & $33(85)$ & $36(92)$ & NS \\
\hline Dyslipidemia, n (\%) & $21(54)$ & $20(51)$ & NS \\
\hline SCORE, \%, median $(01 ; 03)$ & $4.0(2.0 ; 9.0)$ & $6.5(3.0 ; 14.5)$ & NS \\
\hline CCA-IMTb, mm, mean (SD) & $0.93(0.13)$ & $0.96(0.16)$ & NS \\
\hline Atherosclerotic plaques in $\mathrm{CCA}^{\mathrm{b}}, \mathrm{n} / \mathrm{N}(\%)$ & $8 / 27(30)$ & $12 / 24(50)$ & NS \\
\hline Radial artery calcifications ${ }^{\mathrm{c}}, \mathrm{n} / \mathrm{N}(\%)$ & $16 / 25(64)$ & $13 / 20(65)$ & NS \\
\hline Serum creatinine, $\mu \mathrm{mol} / \mathrm{l}$, median $(01 ; 03)$ & $403(314 ; 512)$ & $452(372 ; 596)$ & NS \\
\hline Uric acid, $\mu \mathrm{mol} / \mathrm{l}$, median $(01 ; 03)$ & $395(285 ; 460)$ & $288(228 ; 369)$ & 0.002 \\
\hline $\mathrm{Ca} \times \mathrm{Pi}, \mathrm{mmol}^{2} / \mathrm{I}^{2}$, median $(01 ; 03)$ & $2.99(2.75 ; 3.70)$ & $3.57(2.88 ; 4.16)$ & NS \\
\hline iPTH, pg/ml, median $(01 ; 03)$ & $279(172 ; 453)$ & $208(152 ; 655)$ & NS \\
\hline OPG, pmol/l, median $(01 ; 03)$ & $5.93(3.66 ; 8.05)$ & $8.58(6.21 ; 13.47)$ & $<0.001$ \\
\hline OPN, ng/ml, median $(01 ; 03)$ & $219(160 ; 344)$ & $356(238 ; 631)$ & 0.003 \\
\hline $\mathrm{OC}, \mathrm{ng} / \mathrm{ml}$, median $(01 ; 03)$ & $42.5(29.8 ; 74.6)$ & $44.1(33.6 ; 70.0)$ & NS \\
\hline Fetuin $A, g / l$, mean (SD) & $0.234(0.041)$ & $0.245(0.066)$ & NS \\
\hline FGF-23, RU/ml, median $(01 ; 03)$ & $522(265 ; 1243)$ & $2529(1006 ; 5066)$ & $<0.001$ \\
\hline Albumin, g/l, mean (SD) & $42.0(4.0)$ & $39.1(5.6)$ & 0.035 \\
\hline Fibrinogen, $\mathrm{g} / \mathrm{l}$, median $(01 ; 03)$ & $4.73(4.34 ; 5.53)$ & $5.50(5.02 ; 5.96)$ & 0.011 \\
\hline hs-CRP, mg/l, median (01; 03) & $3.08(1.26 ; 8.47)$ & $12.20(6.68 ; 28.85)$ & $<0.001$ \\
\hline IL-6, pg/ml, median (01; 03) & $2.60(1.52 ; 5.61)$ & $5.58(3.63 ; 8.67)$ & $<0.001$ \\
\hline IL-18, ng/ml, median (01; 03) & $607(461 ; 775)$ & $702(505 ; 990)$ & NS \\
\hline TNFR2, $\mu \mathrm{g} / \mathrm{ml}$, median $(01 ; 03)$ & $11.7(9.3 ; 16.5)$ & $16.6(13.7 ; 23.4)$ & $<0.001$ \\
\hline TGF- $\beta_{1}, \mu \mathrm{g} / \mathrm{ml}$, median $(01 ; 03)$ & $5.01(3.46 ; 6.44)$ & $5.83(4.41 ; 8.00)$ & 0.043 \\
\hline HGF, ng/ml, median $(01 ; 03)$ & $1.95(1.59 ; 2.53)$ & $2.99(2.43 ; 4.02)$ & $<0.001$ \\
\hline TM, ng/ml, median (01; 03) & $15.8(13.6 ; 19.2)$ & $17.5(14.9 ; 22.5)$ & 0.035 \\
\hline SDF-1 $\alpha, \mathrm{ng} / \mathrm{ml}$, median $(01 ; 03)$ & $2.94(2.63 ; 3.37)$ & $3.39(2.83 ; 3.62)$ & 0.024 \\
\hline
\end{tabular}

a Data provided for patients dialyzed at baseline

b Data provided for patients with available results of common carotid artery ultrasound (ie, 27 patients with PTX3 $\leq 1.43 \mathrm{ng} / \mathrm{ml}$ and 24 with PTX3 >1.43 ng/ml)

c Data provided for patients with available results of radial artery alizarin-red staining (ie, 25 patients with PTX3 $\leq 1.43 \mathrm{ng} / \mathrm{ml}$ and 20 with PTX3 $>1.43 \mathrm{ng} / \mathrm{ml}$ )

Abbreviations: BMI, body mass index; Ca, calcium; CCA-IMT, common carotid artery intima-media thickness; FGF-23, fibroblast growth factor 23; HGF, hepatocyte growth factor; hs-CRP, high-sensitivity C-reactive protein; IL-6, interleukin 6; IL-18, interleukin 18; iPTH, intact parathyroid hormone; NS, nonsignificant, OC, osteocalcin; OPG, osteoprotegerin; OPN, osteopontin; Pi, phosphate; PTX3, pentraxin 3; SCORE, Systematic Coronary Risk Evaluation; TGF- $\beta_{1}$, transforming growth factor $\beta_{1}$; SDF-1 $\alpha$, stromal cell-derived factor 1 $\alpha$; TM, thrombomodulin; TNFR2, tumor necrosis factor receptor 2

Association between pentraxin 3 and mortality During the 5-year follow-up, 27 patients (35\%) died, of whom 25 died due to $\mathrm{CV}$ causes. The causes of death were as follows: HF in 11 patients, MI in 11, stroke in 3, and neoplasm in 2. During the follow-up, all patients who had not been dialyzed at baseline began HD therapy and 14 patients (18\%) underwent renal transplantation. The median follow-up time in the studied group was 48 months (23; 56 months), and the median survival time was not reached (Q1: 25 months). All-cause and CV mortality was higher in the group of patients with serum PTX3 concentrations above the median value ( 18 vs 9 deaths; 17 vs 8 deaths from CV causes; FIGURE 2); the hazard ratio for death was 2.13 (95\% CI, 1.01-4.49; $P=0.048$ ) in patients with PTX3 levels above the median value as compared with those with PTX3 levels below 
FIGURE 1 Sections of radial arteries stained with alizarin red, demonstrating low degree of radial artery calcification (RAC) (A) and high degree of $R A C(B)$.
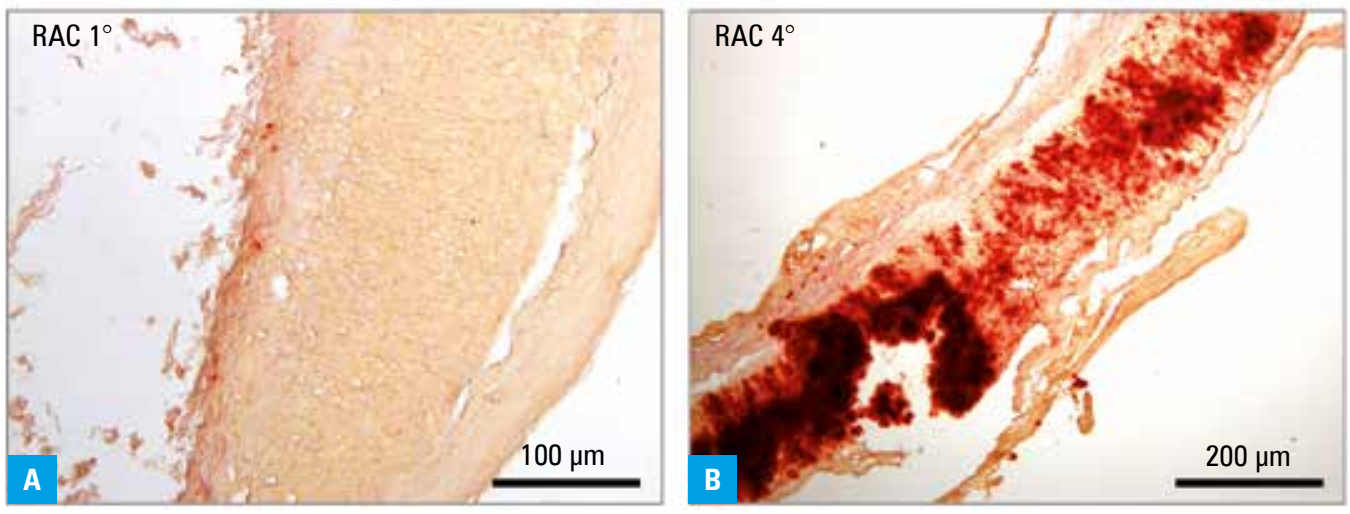

FIGURE 2

Cardiovascular mortality among patients with pentraxin 3 (PTX3) above (blue line) and below (black line) the median value of $1.43 \mathrm{ng} / \mathrm{ml}$. The lower quartile of survival time was 24 months in the group of patients with PTX3 above the median (median survival not reached) and was not reached in the group with PTX3 below the median value.

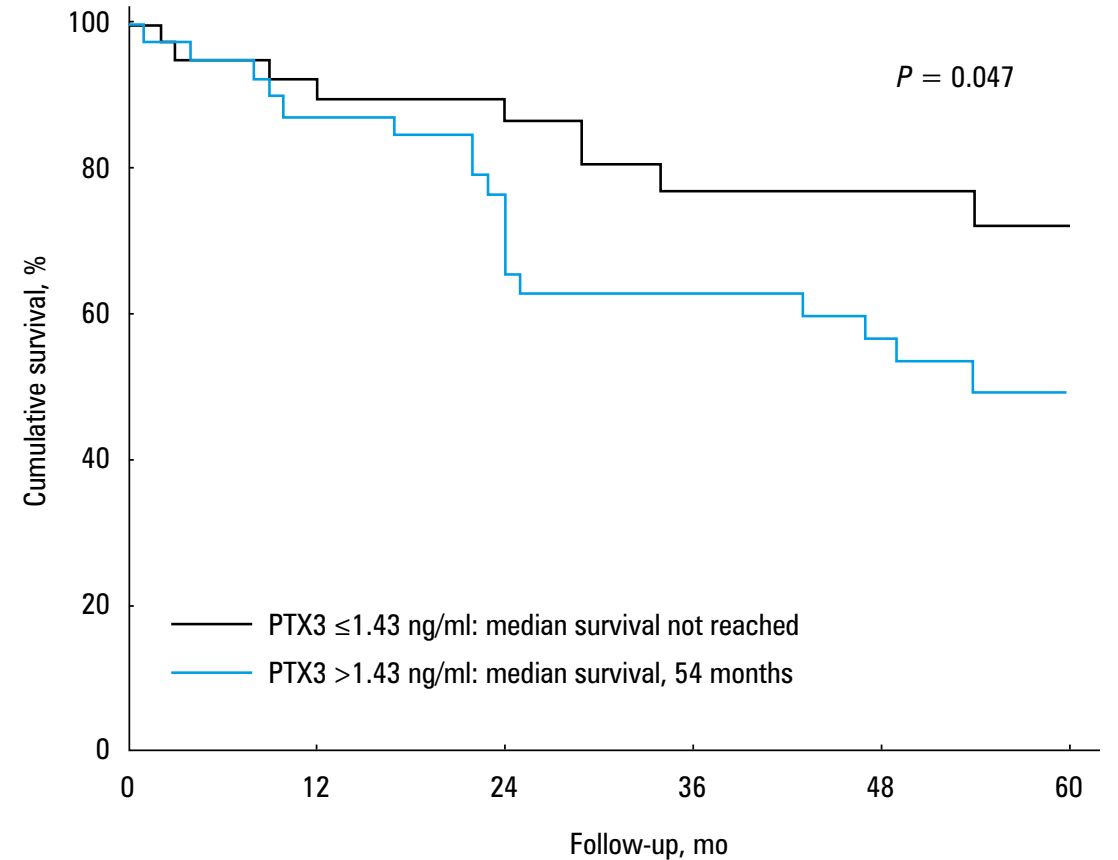

TABLE 2 Simple correlations between $\log (\mathrm{PTX} 3)$ and other studied variables

\begin{tabular}{lll} 
Variable & $r$ & $P$ value \\
Age & 0.25 & 0.028 \\
\hline Log(BMI) & -0.25 & 0.024 \\
\hline Dialysis treatment duration & 0.37 & 0.001 \\
\hline Log(serum creatinine) & 0.23 & 0.039 \\
\hline Log(hs-CRP) & 0.42 & $<0.001$ \\
\hline Log(IL-6) & 0.46 & 0.001 \\
\hline Log(IL-18) & 0.30 & 0.007 \\
\hline Log(TNFR2) & 0.44 & $<0.001$ \\
\hline Log(TGF- $\left.\beta_{1}\right)$ & 0.31 & 0.006 \\
\hline Log(HGF) & 0.49 & $<0.001$ \\
\hline Log(Ca $\times$ Pi) & 0.25 & 0.030 \\
\hline Log(OPG) & 0.51 & $<0.001$ \\
\hline Log(OPN) & 0.31 & 0.006 \\
\hline Log(FGF-23) & 0.46 & $<0.001$ \\
\hline Log(TM) & 0.26 & 0.025 \\
\hline SDF-1a & 0.29 & 0.012 \\
\hline Uric acid & -0.35 & 0.002 \\
\hline
\end{tabular}

Abbreviations: see TABLE 1 the median value. In an unadjusted Cox regression analysis, PTX3 concentrations significantly predicted all-cause and CV mortality (TABLE 3). This association was confirmed after adjustment for dialysis status at baseline and for other known predictors of mortality such as age, serum OPG and hs-CRP concentrations, the presence of radial artery calcifications, and the value of CCA-IMT (TABLE 3). Of the other inflammatory markers, only hs-CRP was significantly associated with CV mortality: hazard ratio, 1.01 (95\% CI, 1.002-1.03) $(P=0.047)$, while no such association was observed for IL-6 $(P=0.6)$, IL-18 $(P=0.9)$, TNFR2 $(P=0.9)$, TGF- $\beta(P=0.4)$, and HGF $(P=0.085)$. However, only PTX3 levels significantly predicted CV mortality in addition to CV risk assessed using the SCORE (TABLE 4).

DISCUSSION To our knowledge, the relationship between PTX3 levels and long-term mortality, the presence of calcification in the media of the radial artery, as well as CCA-IMT in patients with CKD has not been studied before. Our study showed that increased PTX3 concentrations 
TABLE 3 Cox regression models for all-cause and cardiovascular mortality.

\begin{tabular}{|c|c|c|c|c|c|c|}
\hline \multirow{2}{*}{$\begin{array}{l}\text { Independent } \\
\text { variable }\end{array}$} & \multicolumn{3}{|c|}{ All-cause mortality } & \multicolumn{3}{|c|}{ Cardiovascular mortality } \\
\hline & Simple model & Multiple model $1^{\mathrm{a}}$ & Multiple model 2a,b & Simple model & Multiple model $1^{\mathrm{a}}$ & Multiple model $2^{\mathrm{a}}$ \\
\hline PTX3, per $1 \mathrm{ng} / \mathrm{ml}$ & $\begin{array}{c}1.28(1.12-1.48) \\
P<0.001\end{array}$ & $\begin{array}{c}1.31(1.06-1.64) \\
P=0.013\end{array}$ & $\begin{array}{c}1.28(1.05-1.55) \\
P=0.012\end{array}$ & $\begin{array}{c}1.28(1.11-1.47) \\
P<0.001\end{array}$ & $\begin{array}{c}1.33(1.07-1.66) \\
P=0.010\end{array}$ & $\begin{array}{c}1.28(1.04-1.57) \\
P=0.017\end{array}$ \\
\hline OPG, per $1 \mathrm{pmol} / \mathrm{l}$ & - & $\begin{array}{c}1.07(0.97-1.19) \\
P=0.2\end{array}$ & - & - & $\begin{array}{l}1.05(0.94-1.17) \\
P=0.3\end{array}$ & - \\
\hline hs-CRP, per $1 \mathrm{mg} / \mathrm{l}$ & - & $\begin{array}{l}1.01(0.99-1.02) \\
P=0.3\end{array}$ & - & - & $\begin{array}{l}1.01(0.99-1.02) \\
P=0.5\end{array}$ & - \\
\hline $\begin{array}{l}\text { Radial artery } \\
\text { calcifications }\end{array}$ & - & - & $\begin{array}{l}1.35(0.36-5.06) \\
P=0.6\end{array}$ & - & - & $\begin{array}{l}1.75(0.40-7.62) \\
P=0.4\end{array}$ \\
\hline $\begin{array}{l}\text { CCA-IMT, per } \\
0.1 \mathrm{~mm}\end{array}$ & - & - & $\begin{array}{l}0.99(0.57-1.64) \\
P=0.9\end{array}$ & - & - & $\begin{array}{c}1.10(0.66-1.86) \\
P=0.7\end{array}$ \\
\hline
\end{tabular}

Data are reported as hazard ratio $(95 \% \mathrm{Cl})$ and $P$ value.

a Multiple models 1 and 2 are adjusted for age and dialysis status at baseline.

b Multiple model 2 was calculated for patients who underwent histological examination of the radial artery and ultrasonography of the common carotid artery $(n=45)$.

Abbreviations: see TABLE 1

TABLE 4 Comparison of high-sensitivity C-reactive protein and pentraxin 3 as predictors of cardiovascular mortality in addition to classic cardiovascular risk factors as assessed using SCORE

\begin{tabular}{lll} 
Independent variable & Model 1 & Model 2 \\
SCORE, per $1 \%$ & $1.06(1.01-1.12) ; P=0.016$ & $1.07(1.02-1.13) ; P=0.006$ \\
\hline PTX3, per $1 \mathrm{ng} / \mathrm{ml}$ & $1.18(1.02-1.37) ; P=0.026$ & - \\
\hline hs-CRP, per $1 \mathrm{mg} / \mathrm{l}$ & - & $1.01(0.99-1.02) ; P=0.3$ \\
\hline
\end{tabular}

Data are reported as hazard ratio $(95 \% \mathrm{Cl})$ and $P$ value.

Abbreviations: See TABLE 1

significantly predicted both all-cause and CV mortality in patients with advanced CKD. So far, Suliman et $\mathrm{al}^{15}$ and Sjoberg et $\mathrm{al}^{16}$ demonstrated that high PTX3 levels in HD patients were independently associated with all-cause mortality. PTX3 has been shown to be a strong predictor of $\mathrm{CV}$ events in advanced $\mathrm{CHF}$. Liu et $\mathrm{al}^{17}$ revealed that plasma PTX3 levels were significantly higher in patients with CHF than in healthy individuals. Interestingly, PTX3 levels increased with advancing class in the New York Heart Association (NYHA) functional classification and could predict future cardiac events in patients with $\mathrm{CHF}$. Moreover, in a multivariate Cox regression analysis, which included well-known clinical and biochemical risk factors for $\mathrm{CHF}$, the plasma levels of PTX3 remained an independent predictor of cardiac events in those patients. In another study, Suzuki et al ${ }^{18}$ found that PTX3 levels increased significantly in patients with $\mathrm{CHF}$ as compared to healthy controls and also increased with advancing NYHA functional class. These authors also demonstrated that the plasma PTX3 level could be a prognostic risk factor in patients with CHF. Similarly, the Controlled Rosuvastatin Multinational Trial in CHF (CORONA, N = 1457 patients) and GISSI-Heart Failure trials $(\mathrm{N}=1233)^{19}$ assessed the correlation between plasma levels of PTX3 and mortality in CHF patients during a 3-month follow-up. Elevated PTX3 levels were associated with a higher risk of all-cause mortality (759 events, 28.3\%), CV mortality (587 patients, 21.9\%), or hospitalization for worsening HF (720 events, 26.9\%). Three-month changes in PTX3 levels were associated with fatal events after adjustment for hs-CRP or N-terminal pro-B-type natriuretic peptide. It is worth noting that in 2 independent randomized clinical trials including patients with CHF, elevated PTX3 levels were consistently associated with fatal outcomes. Patients with acute coronary syndrome also showed high levels of PTX3. Mielva et $\mathrm{al}^{20}$ recruited 873 patients presenting with chest pain for a prospective observational study. During a 7-year follow-up, 332 patients died and 203 suffered from troponin T-positive nonfatal cardiac event. In a multivariate analysis, PTX3 levels above the median value of $5.88 \mathrm{ng} / \mathrm{ml}$ ndependently predicted mortality. Similarly, in our study all-cause mortality was higher in the group of patients with serum PTX3 concentrations above the median value. The risk of death at 5 years in these patients was more than twice as high as that for patients with PTX3 levels below the median value. In patients with PTX3 above $1.43 \mathrm{ng} / \mathrm{ml}$, the median survival was 54 months. The baseline PTX3 concentration predicted mortality not only in an unadjusted analysis, but also in the models adjusted for important predictors of death. 
In the Multi-Ethnic Study of Atherosclerosis (MESA), ${ }^{21}$ PTX3 was not associated with the risk of stroke, $\mathrm{CV}$ death, or non-CV death but it was related to increased risk of $\mathrm{MI}, \mathrm{CVD}$, and $\mathrm{CHF}$ events. The authors showed that in a population-based study of men and women from 4 ethnic groups (2880 participants with a follow-up of 8.5 years) who were free of clinical CVD these associations were independent of hs-CRP levels. On the other hand, in the early Cardiovascular Health Study, ${ }^{2}$ conducted by the same authors in the group of over 1500 participants, PTX3 levels were significantly associated with the risk of $\mathrm{CV}$ and all-cause death. In that study, PTX3 levels were not associated either with incidents of angina, MI, or stroke or with a combined endpoint of angina, MI, stroke, and CV death. Similarly to the MESA study, the addition of hs-CRP to the model had no significant effect on risk prediction by PTX3, although of all other inflammatory markers studied only hs-CRP was significantly associated with CV mortality. However, only PTX3 significantly predicted CV mortality in addition to $C V$ risk assessed using the SCORE. Likewise, there was no significant interaction between PTX3 and hs-CRP levels for any outcome. This might confirm our assumption that PTX3 reflects different aspects of atherosclerosis-related inflammation than hs-CRP and may provide additional insight into the development of vascular damage in patients with CKD. It is also consistent with our hypothesis that baseline PTX3 concentrations as an indicator of local vascular damage response levels could provide more valuable information on the development and progression of atherosclerosis than nonspecific markers such as hs-CRP. PTX3 thus appears to be a more specific marker for local vascular inflammation but not for systemic inflammation.

So far, the PTX3 concentration has been proposed to be a risk marker for coronary artery disease, ${ }^{22} \mathrm{MI},{ }^{23}$ and aortic valve calcification. ${ }^{11}$ The study of Hudzik et al ${ }^{24}$ showed that the PTX3 level was a marker of advanced coronary artery disease. Similarly as in our study, PTX3 levels were correlated with other $\mathrm{CV}$ risk factors such as hs-CRP. Moreover, in our study PTX3 appeared to be a marker of the risk of $C V$ death (related to HF, MI, or stroke) in patients with CKD. The PTX3 level was independently associated with CV mortality, but there was no association with elevated CCA-IMT, an early precursor of atherosclerosis, and there was no relationship with severity of calcifications in the media of the radial artery. This could be due to a relatively small number of patients from whom these data were available. However, the results of our study are consistent with those reported by Knoflach et $\mathrm{al}^{25}$ for the Atherosclerosis Risk Factors in Male Youngsters (ARMY) and the Atherosclerosis Risk Factor in Female Youngsters (ARFY) studies, where no relationship was found between the intima-media ratio of large arteries (CCA-IMT and femoral artery IMT) and elevated concentrations of PTX3.
These studies, however, demonstrated an association of high PTX3 levels with late CV complications of atherosclerosis such as angina, peripheral artery disease, MI, and stroke.

In agreement with previous studies, ${ }^{19,21}$ our patients with higher PTX3 levels were older and had lower BMI. Bosutti et $\mathrm{al}^{26}$ reported an inverse association of PTX3 with fat mass and hypothesized that nutrition can play a role in the regulation of PTX3 levels. Similarly, in the study of Sjoberg et al, ${ }^{16}$ patients with high stable PTX3 levels had significantly lower albumin levels and $\mathrm{BMI}$, lean BMI, and fat BMI, as well as higher Davies score ${ }^{27}$ as compared to those with stable low PTX3 levels, indicating that patients with elevated PTX3 levels suffered from protein-energy wasting and had more comorbidities. A possible explanation for this is advanced state of inflammation, being a part of the malnutrition-inflammation-atherosclerosis syndrome in patients with CKD, which decreases the BMI.

In our study, in line with other reports, ${ }^{28,29} \mathrm{pa}-$ tients with higher concentrations of PTX3 had more advanced renal failure and more severe inflammation and endothelial dysfunction. Tong et $\mathrm{al}^{28}$ showed that patients (stage 3-5 CKD) with protein-energy wasting, inflammation, and $\mathrm{CV}$ disease had higher concentrations of PTX3 than their healthy counterparts. Moreover, patients with high PTX3 levels had higher all-cause but not CV mortality. Similarly to the results reported by Suliman et al, ${ }^{15}$ in our study the PTX3 concentration was positively correlated with hs-CRP and IL-6 levels, and negatively with serum albumin levels. Release of proinflammatory cytokines and metabolic abnormalities associated with systemic inflammation belong to principal mechanisms leading to endothelial dysfunction in patients with uremia. Local ischemia damages endothelial cells and stimulates production of long PTX in the vasculature, and PTX3 is rapidly detectable in the systemic circulation. ${ }^{1,3,4}$

We demonstrated a significant correlation of PTX3 concentrations with TM and SDF- $1 \alpha$, biochemical markers of endothelial activation, which leads to changes in the vessel wall. Vascular calcifications in patients with uremia are located both in the intima and in the media. ${ }^{30,31}$ In the present study, an interesting finding is the correlation between PTX3 levels and markers of calcification: OPG, OPN, and FGF-23. Furthermore, elevated OPG levels predicted high PTX3 levels independently of age, time on dialysis, inflammation, and endothelial damage. PTX3 concentrations significantly correlated not only with OPG but also with TNFR2. According to our previous reports, ${ }^{31,32}$ OPG may be an indicator of inflammation, atherosclerosis, and medial artery calcification. OPG may also participate in the calcific degeneration of the heart valves. ${ }^{33}$

This study has demonstrated that increased PTX3 concentrations significantly predict CV mortality in patients with advanced $C K D$ regardless of serum OPG and hs-CRP levels, the presence of 
radial artery calcifications, the value of CCA-IMT, age, and dialysis status at baseline. On the basis of the earlier reports and the present results, it appears that the baseline measurement of serum PTX3 levels should be considered as a routine, noninvasive tool in the evaluation of $\mathrm{CV}$ risk at the initiation of renal replacement therapy. The pathogenetic role of PTX3 in CVD remains unclear and requires further research. However, although limited by a relatively low number of patients, our study suggests that PTX3 might be a useful marker of risk for cardiorenal syndrome and that assessment of PTX3 concentrations could substantially improve the risk stratification of patients with advanced CKD.

In conclusion, we postulate that PTX3 might be an early biomarker of $\mathrm{CV}$ mortality in patients with advanced $C K D$, yet before the increase in the levels of a specific marker for systemic inflammation such as hs-CRP. The prognostic value of the initial measurement of PTX3 concentrations for assessing CV risk in CKD could contribute to early prevention and reduction of mortality in this population.

Acknowledgments We are grateful to all patients who participated in this study. Sources of funding: Financial support was provided by a statutory grant K/ZDS/000 597 from Jagiellonian University Medical College (to KK).

Contribution statement $\mathrm{MK}$ and $\mathrm{KK}$ conceived the idea for the study and were the major contributors to study design, coordination, interpretation of results, and statistical analysis. MG performed histological examinations. PD performed statistical analysis. KW participated in the design of the study. AD and JAL analyzed the data and participated in the design. WS participated in the design and coordination of the study. All authors were involved in data collection. All authors edited and approved the final version of the manuscript.

\section{REFERENCES}

1 Turkmen K, Erdur FM, Guney I, et al. Relationship between plasma pentraxin-3, neutrophil-to-lymphocyte ratio, and atherosclerosis in renal transplant patients. Cardiorenal Med. 2012; 4: 298-307.

2 Jenny NS, Arnold AM, Kuller LH, et al. Associations of pentraxin 3 with cardiovascular disease and all-cause death: the Cardiovascular Health Study. Arterioscler Thromb Vasc Biol. 2009; 29: 594-599.

3 Bonacina F, Baragetti A, Catapano AL, Norata GD. Long pentraxin 3 experimental and clinical relevance in cardiovascular diseases. Mediators Inflamm. 2013; 2013: 725102. doi:10.1155/2013/725102

4 Witasp A, Ryden M, Carrero JJ, et al. Elevated circulating levels and tis sue expression of pentraxin 3 in uremia: a reflection of endothelial dysfunction. PlosOne. 2013; 8: e63 493. doi:10.1371/journal.pone.0063493

5 Fornai F, Carrizzo A, Forte M, et al. The inflammatory protein Pentraxin 3 in cardiovascular disease. Immunn Ageing. 2016; 13. doi:10.1186/ s12 979-016-0080-1

6 Vilahur G, Badimon L. Biological actions of pentraxins. Vascul Pharmacol. 2015; 73: 38-44.

7 Kocyigit I, Eroglu E, Orscelik 0, et al. Pentraxin 3 as a novel bio-marke of inflammation and endothelial dysfunction in autosomal dominant polycystic kidney disease. J Nephrol. 2014; 27: 181-186.

8 Ishino M, Shishido T, Suzuki S, et al. Deficiency of long pentraxin PTX3 promoted neointimal hyperplasia after vascular injury. J Atheroscler Thromb. $2015 ; 22: 372-378$
Sjoberg B, Oureshi AR, Heimburger 0 , et al. Association between levels of pentraxin 3 and incidence of chronic kidney disease in the elderly. J Intern Med. 2016; 279: 173-179.

10 Zhou Y, Ni Z, Zhang J, et al. Plasma pentraxin 3 may be a better mark er of peripheral artery disease in hemodialysis patients than C-reactive protein. Vasc Med. 2013; 2: 85-91.

11 Norimatsu K, Miura SI, Suematsu Y, et al. Association between pentraxin 3 levels and aortic valve calcification. J Cardiol. 2015; 68: 76-82.

12 Heart Score. www. HeartScore.org/pl. Accessed December 30, 2016.

13 Perk J, De Backer G, Gohlke H, et al. European Guidelines on CVD Prevention in Clinical Practice (2012). The Fifth Joint Task Force of the Europe an Society of Cardiology and Other Societies on Cardiovascular Disease Prevention in Clinical Practice (constituted by representatives of nine societies and by invited experts). Eur Heart J. 2012; 33: 1635-1701.

14 Levey AS, Coresh J, Greene T, et al. Chronic Kidney Disease Epidemioogy Collaboration Expressing the Modification of Diet in Renal Disease Study equation for estimating glomerular filtration rate with standardized serum creatinine values. Clin Chem. 2007; 4: 766-772

15 Suliman ME, Qureshi AR, Carrero JJ, et al. The long pentraxin PTX-3 in prevalent hemodialysis patients: associations with comorbidities and mortality. OJM. 2008; 101: 397-405.

16 Sjoberg B, Snaedal S, Stenvinkel P, et al. Three-month variation of plas ma pentraxin 3 compared with C-reactive protein, albumin and homocysteine levels in haemodialysis patients. Clin Kidney J. 2014; 4: 373-379.

17 Liu H, Guo X, Yao K, et al. Pentraxin-3 predicts long-term cardiac events in patients with chronic heart failure. Biomed Res Int. 2015; 2015 817615.

18 Suzuki S, Takeishi Y, Niizeki T, et al. Pentraxin 3, a new marker for vascular inflammation, predicts adverse clinical outcomes in patients with heart failure. Am Heart J. 2008; 155: 75-81.

19 Latini R, Gullestad L, Masson S, et al. Pentraxin-3 in chronic heart failure: the CORONA and GISSI-HF trials. Eur J Heart Fail. 2012; 9: 992-999.

20 Mjelva R, Ponitz V, Brugger-Andersen T, et al. Long-term prognostic utility of pentraxin 3 and D-dimer as compared to high-sensitivity C-reactive protein and B-type natriuretic peptide in suspected acute coronary syndrome. Eur J Prev Cardiol. 2016; 11: 1130-1140.

21 Jenny NS, Blumenthal RS, Kronmal RA, et al. Associations of pentraxin 3 with cardiovascular disease: the Multi-Ethnic Study of Atherosclerosis J Thromb Haemost. 2014; 6: 999-1005.

22 Nerkiz P, Doganer YC, Aydogan U, et al. Serum pentraxin-3 level in patients who underwent coronary angiography and relationship with coronary atherosclerosis. Med Princ Pract. 2015; 24: 369-375.

23 Matsui $S$, Ishii J, Kitagawa F, et al. Pentraxin 3 in unstable angina and non-ST-segment elevation myocardial infarction. Atherosclerosis. 2010; 210: $220-225$

24 Hudzik B, Danikiewicz A, Szkodzinski J, et al. Pentraxin-3 concentrations in stable coronary artery disease depend on the clinical presentation. Eur Cytokine Netw. 2014; 3: 41-45.

25 Knoflach M, Kiechl S, Mantovani A, et al. Pentraxin-3 as a marker of advanced atherosclerosis results from the Bruneck, ARMY and ARFY Studies. PLosOne. 2012; 7: e31474. doi:10.1371/journal.pone.0031474

26 Bosutti A, Malaponte G, Zanetti M, et al. Calorie restriction modulates inactivity-induced changes in the inflammatory markers $\mathrm{C}$-reactive protein and pentraxin-3. J Clin Endocrinol Metab. 2008; 93: 3226-3229.

27 Davies SJ, Phillips L, Naish PF, Russell Gl. Quantifying comorbidity in peritoneal dialysis patients and its relationship to other predictors of survival. Nephrol Dial Transplant. 2002; 17: 1085-1092.

28 Tong $\mathrm{M}$, Carrero JJ, Qureshi AR, et al. Plasma pentraxin 3 in patients with chronic kidney disease: associations with renal function, protein-energy wasting, cardiovascular disease, and mortality. Clin J Am Soc Nephrol. 2007; 5: 889-897.

29 Nishi K, Imamura T, Kitamura K, et al. Associations of plasma pentraxin 3 and monocyte chemoattractant protein-1 concentrations with cardiovascular disease in patients with chronic kidney disease. Ren Fail. 2011; 33: 398-404.

30 Kurnatowska I, Grzelak P, Masajtis-Zagajewska A, et al. Effect of vita$\min \mathrm{K} 2$ on progression of atherosclerosis and vascular calcification in nondialyzed patients with chronic kidney disease stages 3-5. Pol Arch Med Wewn. 2015; 125: 631-640.

31 Janda K, Krzanowski M, Gajda M, et al. Cardiovascular risk in chronic kidney disease patients: intima-media thickness predicts the incidence and severity of histologically assessed medial calcification in radial arteries. BMC Nephrol. 2015; 16: 78. doi:10.1186/s12 882-015-0067-8

32 Janda K, Krzanowski M, Chowaniec E, et al. Osteoprotegerin as a marker of cardiovascular risk in patients on peritoneal dialysis. Pol Arch Med Wewn. 2013; 123: 149-155.

33 Lis GJ, Czubek U, Jasek-Gajda E, et al. Influence of osteoclasts and osteoprotegerin on the mode of calcific degeneration of aortic valves. Pol Arch Med Wewn. 2016; 126: 149-158. 